\title{
Reduced-Order Model Construction Procedure for Robust Mistuning Identification of Blisks
}

\author{
Andrew C. Madden, $*$ Matthew P. Castanier, $₫$ and Bogdan I. Epureanu $\stackrel{\ddagger}{ \pm}$ \\ University of Michigan, Ann Arbor, Michigan 48109-2125
}

DOI: $\underline{10.2514 / 1.37314}$

\begin{abstract}
The reduced-order modeling of integrally bladed disks for predicting the mistuned vibration response has been well studied and understood. For solving a direct vibration problem, adding modes to the modeling basis improves the accuracy of the reduced-order model with respect to the parent finite element model. In contrast, when solving an inverse problem for system identification, adding modes to the reduced-order model while using the same measurements may actually reduce its accuracy. This is especially true for solving inverse problems related to the identification of blade mistuning parameters, because the characteristics of the selected system modes for the reduced-order model may not match the assumptions used in the mistuning modeling approach. In this work, a procedure is introduced for constructing a reduced-order model referred to as the inverse reduced-order model that is well suited for solving the mistuning identification inverse problem. First, a quantitative metric is defined to characterize and rank the tuned-system modes with respect to their suitability for constructing inverse reducedorder models. Then, the direct problem is solved using a larger direct reduced-order model with prescribed mistuning to interrogate and validate the performance of various inverse reduced-order models as modes are added. This enables the automated construction of suitable inverse reduced-order models and improves the overall accuracy and robustness of mistuning identification.
\end{abstract}

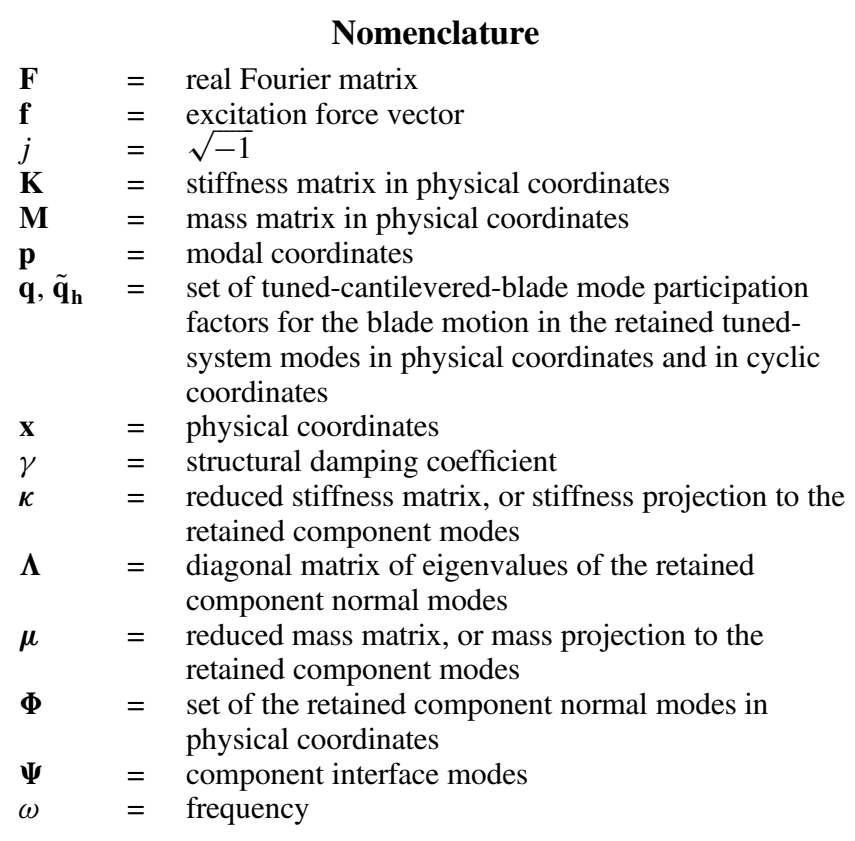

Subscripts

$A=$ active degree of freedom

Presented as Paper 2332 at the 49th AIAA SDM Conference, Chicago, 710 April 2008; received 26 February 2008; revision received 17 March 2008; accepted for publication 25 April 2008. Copyright () 2008 by Andrew C. Madden, Matthew P. Castanier, and Bogdan I. Epureanu. Published by the American Institute of Aeronautics and Astronautics, Inc., with permission. Copies of this paper may be made for personal or internal use, on condition that the copier pay the $\$ 10.00$ per-copy fee to the Copyright Clearance Center, Inc., 222 Rosewood Drive, Danvers, MA 01923; include the code 0001-1452/ $08 \$ 10.00$ in correspondence with the CCC.

${ }^{*}$ Graduate Student Research Assistant, Department of Mechanical Engineering. Student Member AIAA.

Associate Research Scientist, Department of Mechanical Engineering. Senior Member AIAA.

${ }^{\ddagger}$ Associate Professor, Department of Mechanical Engineering. Member AIAA.

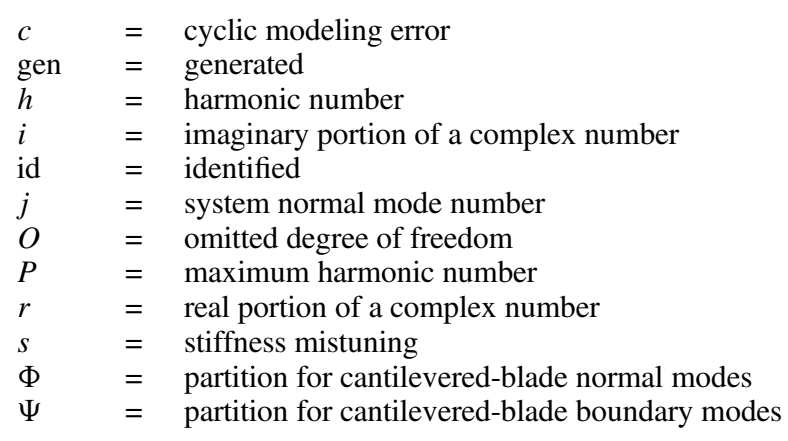

Superscripts

$\mathrm{cb}=$ cantilevered blade

cyc $=$ cyclic modeling error coordinates

mist $=$ stiffness mistuning coordinates

$s \quad=\quad$ tuned system

$\delta=$ mistuning component, or assembly of mistuning components

\section{Introduction}

Q MALL deviations in the structural properties of the blades in an $\boldsymbol{N}$ otherwise cyclically symmetric bladed disk can result in significant changes to the forced response behavior of the structure. These small blade-to-blade deviations, called mistuning, can arise due to reasons such as manufacturing tolerances, general wear over the life cycle, and damage. Mistuning has been shown to increase the forced response for a bladed disk, which can be a concern for high cycle fatigue [1]. Furthermore, due to mistuning, the cyclic symmetry of the system is destroyed along with the possibility of using efficient cyclic symmetry solvers to predict the vibration response. As a result, a large body of research exists on constructing reduced-order models (ROMs) of mistuned bladed disk vibration based on tuned-system and/or component modes [2-8].

With the capability of modeling and studying mistuning of bladed disks in place, several recent research efforts have focused on the identification of the mistuning parameters using experimentally measured system response data. Such system-based mistuning identification procedures are essential in the case of bladed disks 
manufactured as a single piece (called blisks), because the blades cannot be separated from the disk and tested individually. Mistuning identification results can also be used for evaluating manufacturing processes and identifying wear and damage during maintenance checks. Work in this area has ranged from simple lumped parameter models $[9,10]$ to more involved reduced-order modeling techniques [11-21]. Judge et al. [11] found that the identified mistuning parameters were sensitive to errors in the finite element model (FEM) or measurement data. Later, Lim et al. [19] found that the identification results showed especially high sensitivity to errors in the tunedsystem eigenvalues for the modes used in the ROM.

Sensitivity to the FEM and measurement data and their use in the ROM is the subject of this work. Pichot et al. [22] recently presented a mistuning identification procedure in which the measured modes were filtered using the best achievable eigenvectors [23] approach to reduce the errors that occur in the measurement data. The work presented here considers the component mode mistuning (CMM) approach to mistuning identification $[19,20]$ and explores ways to enhance the procedure given the modeling technique and the limitations of the experimental portion of the approach. To select tuned-system normal modes that will best fit the assumptions of the CMM approach, a parameter based on cantilevered-blade participation factors and blade-disk interface motion is introduced. Using modes selected as favorable for the ROM according to this parameter can result in increased accuracy for the mistuning identification procedure. In this work, a procedure is introduced for constructing a ROM referred to as an inverse ROM (or IROM) that is well suited for solving the mistuning identification inverse problem. Also, a representation of the mode shapes using a limited number of measured degrees of freedom (DOF) is accounted for because of the likely possibility that the corresponding modal matrix is rank deficient. The limitations for the types of mode shapes that can be used are discussed. Finally, a method is presented that can be used to determine a suitable IROM size. By using an assumed mistuning pattern, a forward problem can be formulated and used to generate surrogate data, which are then used to identify the mistuning in the inverse problem and, thus, assess the accuracy of the IROM.

\section{Theory}

A. Background: Mistuning Identification and Model Updating Based on the CMM Method

Consider the equations of motion in the frequency domain for an elastic structure with structural damping, expressed as

$$
-\omega^{2} \mathbf{M x}+(1+j \gamma) \mathbf{K} \mathbf{x}=\mathbf{f}
$$

If the structure of interest is a mistuned bladed disk, or blisk, the CMM method developed by Lim et al. [7,8] can be implemented. The CMM procedure treats the tuned system as a free-interface component and the mistuned portions of the system as fixed-interface components. Using component mode synthesis (CMS) [24,25], the following is obtained for the tuned system:

$$
\begin{aligned}
& \mu^{s}=\left[\begin{array}{cc}
\mathbf{I} & \boldsymbol{\Phi}^{s^{T}} \mathbf{M}^{s} \boldsymbol{\Psi}^{s} \\
\boldsymbol{\Psi}^{s^{T}} \mathbf{M}^{s} \boldsymbol{\Phi}^{s} & \boldsymbol{\Psi}^{s^{T}} \mathbf{M}^{s} \boldsymbol{\Psi}^{s}
\end{array}\right] \\
& \kappa^{s}= {\left[\begin{array}{cc}
\boldsymbol{\Lambda}^{s} & \boldsymbol{\Phi}^{s^{T}} \mathbf{K}^{s} \boldsymbol{\Psi}^{s} \\
\boldsymbol{\Psi}^{s^{T}} \mathbf{K}^{s} \boldsymbol{\Phi}^{s} & \boldsymbol{\Psi}_{A}^{s}
\end{array}\right] } \\
& \mathbf{x}^{s}=\left[\begin{array}{ll}
\boldsymbol{\Phi}_{O}^{s} & \boldsymbol{\Phi}_{O}^{s} \\
\boldsymbol{\Phi}_{A}^{s} & \boldsymbol{\Phi}_{A}^{s}
\end{array}\right]\left\{\begin{array}{l}
\mathbf{p}_{\Phi}^{s} \\
\mathbf{p}_{\Psi}^{s}
\end{array}\right\}
\end{aligned}
$$

where $\mu^{s}$ and $\kappa^{s}$ are the reduced mass and stiffness matrices of the tuned system, $\mathbf{p}_{\Phi}^{s}$ and $\mathbf{p}_{\Psi}^{s}$ are modal coordinates, $O$ and $A$ refer to omitted and active (where mistuning exists) DOF, respectively, and $s$ denotes the tuned system. Also, $\boldsymbol{\Phi}^{s}$ and $\boldsymbol{\Psi}^{s}$ are the tuned-system normal modes and constraint modes corresponding to mistuned DOF, respectively, whereas $\mathbf{M}^{s}$ and $\mathbf{K}^{s}$ are the tuned mass and stiffness matrices. The mistuned portions are represented with constraint modes because all of the mistuning DOF are considered interface DOF so that

$$
\begin{aligned}
\mu^{\delta} & =\mathbf{M}^{\delta} \\
\kappa^{\delta} & =\mathbf{K}^{\delta} \\
\mathbf{x}^{\delta} & =\mathbf{x}_{\Psi}^{\delta}
\end{aligned}
$$

Here, $\delta$ denotes the mistuned portion of the system and $\Psi$ indicates the modal coordinates associated with the interface DOF. The CMS synthesized equations follow as

$$
\begin{aligned}
& \mu_{\mathrm{CMS}}^{\mathrm{syn}}=\mu^{s}+\left[\begin{array}{ll}
\boldsymbol{\Phi}_{A}^{s^{T}} \mathbf{M}^{\delta} \boldsymbol{\Phi}_{A}^{s} & \boldsymbol{\Phi}_{A}^{s^{T}} \mathbf{M}^{\delta} \boldsymbol{\Psi}_{A}^{s} \\
\boldsymbol{\Psi}_{A}^{s^{T}} \mathbf{M}^{\delta} \boldsymbol{\Phi}_{A}^{s} & \boldsymbol{\Psi}_{A}^{s^{T}} \mathbf{M}^{\delta} \boldsymbol{\Psi}_{A}^{s}
\end{array}\right] \\
& \kappa_{\mathrm{CMS}}^{\mathrm{syn}}=\kappa^{s}+\left[\begin{array}{ll}
\boldsymbol{\Phi}_{A}^{s^{T}} \mathbf{K}^{\delta} \boldsymbol{\Phi}_{A}^{s} & \boldsymbol{\Phi}_{A}^{s^{T}} \mathbf{K}^{\delta} \boldsymbol{\Psi}_{A}^{s} \\
\boldsymbol{\Psi}_{A}^{s^{T}} \mathbf{K}^{\delta} \boldsymbol{\Phi}_{A}^{s} & \boldsymbol{\Psi}_{A}^{s^{T}} \mathbf{K}^{\delta} \boldsymbol{\Psi}_{A}^{s}
\end{array}\right] \\
& \mathbf{p}_{\mathrm{CMS}}^{\mathrm{syn}}=\left\{\begin{array}{l}
\mathbf{p}_{\Phi}^{s} \\
\mathbf{p}_{\Psi}^{s}
\end{array}\right\}
\end{aligned}
$$

Next, one assumes that the tuned-system modes of interest are in a small frequency range. According to work by Yang and Griffin $[5,26]$, this suggests that the mistuned normal modes are also in a small frequency band and, therefore, can be represented by a small set of tuned-system normal modes in the frequency range of interest. This implies that other normal modes and static modes can be ignored. Equation (4) leads to a reduced-order formulation expressed as

$$
\begin{aligned}
\mu^{\mathrm{syn}} & =\mathbf{I}+\boldsymbol{\Phi}_{A}^{s^{T}} \mathbf{M}^{\delta} \boldsymbol{\Phi}_{A}^{s} \\
\kappa^{\mathrm{syn}} & =\boldsymbol{\Lambda}^{s}+\boldsymbol{\Phi}_{A}^{s^{T}} \mathbf{K}^{\delta} \boldsymbol{\Phi}_{A}^{s} \\
\mathbf{p}^{\mathrm{syn}} & =\mathbf{p}_{\Phi}^{s}=\mathbf{p}
\end{aligned}
$$

Combining Eq. (1) with Eq. (ㅁ) and neglecting mass mistuning $\left(\mathbf{M}^{\delta}\right)$ yields

$$
-\omega^{2} \mathbf{p}+(1+j \gamma)\left[\boldsymbol{\Lambda}^{s}+\boldsymbol{\Phi}^{s^{T}} \mathbf{K}^{\delta} \boldsymbol{\Phi}^{s}\right] \mathbf{p}=\boldsymbol{\Phi}^{s^{T}} \mathbf{f}
$$

In Lim et al. [19,27], an additional term was added to this equation to account for the difference between the parent tuned-system FEM and the virtual tuned system of an actual bladed disk. This term allows the tuned FEM to be updated using the mistuning procedure to more closely match the tuned portion of the actual bladed disk being examined. Adding this term to Eq. (ㅁ), one obtains

$$
\begin{aligned}
& -\omega^{2} \mathbf{p}+(1+j \gamma)\left[\boldsymbol{\Lambda}^{s}+\boldsymbol{\Phi}^{s^{T}}\left(\mathbf{K}^{\delta, s}+\mathbf{K}^{\delta}\right) \boldsymbol{\Phi}^{s}\right] \mathbf{p}=\boldsymbol{\Phi}^{s^{T}} \mathbf{f} \\
& -\omega^{2} \mathbf{p}+(1+j \gamma)\left[\boldsymbol{\Lambda}^{s}+\boldsymbol{\Lambda}^{\delta, s}+\boldsymbol{\Phi}^{s^{T}} \mathbf{K}^{\delta} \boldsymbol{\Phi}^{s}\right] \mathbf{p}=\mathbf{f}^{s}
\end{aligned}
$$

where $\boldsymbol{\Lambda}^{\delta, s}$ is the matrix of deviations of the system eigenvalues from those assumed and $\mathbf{K}^{\delta, s}$ is the deviation of the nominal tuned-system stiffness matrix from that of the actual tuned system. The term $\boldsymbol{\Phi}^{s^{T}} \mathbf{K}^{\delta} \boldsymbol{\Phi}^{s}$ corresponds to the mistuned portion of the stiffness matrix. It should be noted that this term is not decoupled/diagonalized using this modal decomposition. To decouple this portion of the equation and to further reduce the model, the blade portion of the system normal modes, $\boldsymbol{\Phi}^{s}$, is represented using a basis of cantilevered-blade normal modes denoted by $\boldsymbol{\Phi}^{\mathrm{cb}}$. Furthermore, in these coordinates, the off-diagonal terms are considered negligible [27]. Assuming that $\boldsymbol{\Phi}^{s}=\boldsymbol{\Phi}^{\mathrm{cb}} \mathbf{q}$, Eq. (7) can be written as

$$
\begin{array}{r}
-\omega^{2} \mathbf{p}+(1+j \gamma)\left[\boldsymbol{\Lambda}^{s}+\boldsymbol{\Lambda}^{\delta, s}+\mathbf{q}^{T} \boldsymbol{\Phi}^{\mathrm{cb}^{T}} \mathbf{K}^{\delta} \boldsymbol{\Phi}^{\mathrm{cb}} \mathbf{q}\right] \mathbf{p}=\mathbf{f}^{s} \\
-\omega^{2} \mathbf{p}+(1+j \gamma)\left[\boldsymbol{\Lambda}^{s}+\boldsymbol{\Lambda}^{\delta, s}+\mathbf{q}^{T} \boldsymbol{\Lambda}^{\delta, \mathrm{cb}} \mathbf{q}\right] \mathbf{p}=\mathbf{f}^{s}
\end{array}
$$

Equation ( $\underline{8})$ can be rearranged as follows:

$$
(1+j \gamma)\left[\boldsymbol{\Lambda}^{\delta, s}+\mathbf{q}^{T} \boldsymbol{\Lambda}^{\delta, \mathrm{cb}} \mathbf{q}\right] \mathbf{p}=\mathbf{f}^{s}+\omega^{2} \mathbf{p}-(1+j \gamma) \boldsymbol{\Lambda}^{s}
$$

or 


$$
\begin{aligned}
& (1+j \gamma)\left[\boldsymbol{\Lambda}^{s}\left(\boldsymbol{\Lambda}^{s^{-1}} \boldsymbol{\Lambda}^{\delta, s}\right)+\mathbf{q}^{T} \boldsymbol{\Lambda}^{\mathrm{cb}}\left(\boldsymbol{\Lambda}^{\mathrm{cb}^{-1}} \boldsymbol{\Lambda}^{\delta, \mathrm{cb}}\right) \mathbf{q}\right] \mathbf{p}=\mathbf{f}^{s} \\
& \quad+\omega^{2} \mathbf{p}-(1+j \gamma) \boldsymbol{\Lambda}^{s}
\end{aligned}
$$

Because $\mathbf{p}$ is a complex quantity, it can be written as $\mathbf{p}=\mathbf{p}_{r}+j \mathbf{p}_{i}$. Substituting this into Eq. (9) and using $(1+j \gamma)\left(\mathbf{p}_{r}+j \mathbf{p}_{i}\right)=\left(\mathbf{p}_{r}-\right.$ $\left.\gamma \mathbf{p}_{i}\right)+j\left(\mathbf{p}_{i}+\gamma \mathbf{p}_{r}\right)$ yields

$$
\begin{aligned}
& {\left[\boldsymbol{\Lambda}^{s}\left(\boldsymbol{\Lambda}^{s^{-1}} \boldsymbol{\Lambda}^{\delta, s}\right)+\mathbf{q}^{T} \boldsymbol{\Lambda}^{\mathrm{cb}}\left(\boldsymbol{\Lambda}^{\mathrm{cb}-1} \boldsymbol{\Lambda}^{\delta, \mathrm{cb}}\right) \mathbf{q}\right]\left[\left(\mathbf{p}_{r}-\gamma \mathbf{p}_{i}\right)+j\left(\mathbf{p}_{i}+\gamma \mathbf{p}_{r}\right)\right]} \\
& \quad=\mathbf{f}^{s}+\omega^{2}\left(\mathbf{p}_{r}+j \mathbf{p}_{i}\right)-\boldsymbol{\Lambda}^{s}\left[\left(\mathbf{p}_{r}-\gamma \mathbf{p}_{i}\right)+j\left(\mathbf{p}_{i}+\gamma \mathbf{p}_{r}\right)\right]
\end{aligned}
$$

Equation (10) can be split into two equations corresponding to real and imaginary parts as

$$
\begin{gathered}
\boldsymbol{\Lambda}^{s}\left(\boldsymbol{\Lambda}^{s^{-1}} \boldsymbol{\Lambda}^{\delta, s}\right)\left(\mathbf{p}_{r}-\gamma \mathbf{p}_{i}\right)+\mathbf{q}^{T} \boldsymbol{\Lambda}^{\mathrm{cb}}\left(\boldsymbol{\Lambda}^{\mathrm{cb}} \mathrm{b}^{-1} \boldsymbol{\Lambda}^{\delta, \mathrm{cb}}\right) \mathbf{q}\left(\mathbf{p}_{r}-\gamma \mathbf{p}_{i}\right)=\mathbf{f}^{s} \\
+\omega^{2} \mathbf{p}_{r}-\boldsymbol{\Lambda}^{s}\left(\mathbf{p}_{r}-\gamma \mathbf{p}_{i}\right), \underbrace{\Lambda}_{\mathbf{a}_{c 1}}\left(\boldsymbol{\Lambda}^{s^{-1}} \boldsymbol{\Lambda}^{\delta, s}\right) \underbrace{\left(\mathbf{p}_{i}+\gamma \mathbf{p}_{r}\right)}_{\mathbf{a}_{c 2}} \\
+\underbrace{\mathbf{q}^{T} \boldsymbol{\Lambda}^{\mathrm{cb}}}_{\mathbf{a}_{s 1}}\left(\boldsymbol{\Lambda}^{\mathrm{cb} b^{-1}} \boldsymbol{\Lambda}^{\delta, \mathrm{cb}}\right) \underbrace{\mathbf{q}\left(\mathbf{p}_{i}+\gamma \mathbf{p}_{r}\right)}_{\mathbf{a} s 2}=\underbrace{\omega^{2} \mathbf{p}_{i}-\boldsymbol{\Lambda}^{s}\left(\mathbf{p}_{i}+\gamma \mathbf{p}_{r}\right)}_{\mathbf{b}}
\end{gathered}
$$

The diagonal matrices associated with quantities to be identified are organized into column vectors and the rest of the equation is reshaped accordingly. The final matrix equation is represented by

$$
\left[\begin{array}{ll}
\mathbf{A}_{c} & \mathbf{A}_{s}
\end{array}\right]\left[\begin{array}{l}
\mathbf{d}^{\mathrm{cyc}} \\
\mathbf{d}^{\mathrm{mist}}
\end{array}\right]=\mathbf{b}
$$

where $\mathbf{d}^{\mathrm{cyc}}=\operatorname{diag}\left[\boldsymbol{\Lambda}^{s-1} \boldsymbol{\Lambda}^{\delta, s}\right]$ and $\mathbf{d}^{\text {mist }}=\operatorname{diag}\left[\boldsymbol{\Lambda}^{\mathrm{cb}}{ }^{-1} \boldsymbol{\Lambda}^{\delta, \mathrm{cb}}\right]$, whereas matrices $\mathbf{A}_{c}$ and $\mathbf{A}_{s}$ are composed of a reorganized version of $\mathbf{a}_{c 1}$ and $\mathbf{a}_{c 2}$ and of $\mathbf{a}_{s 1}$ and $\mathbf{a}_{s 2}$.

\section{B. Selection Ratio}

The CMM approach to mistuning identification presented in the previous section assumes that the system modes have certain properties typically present in blisks in frequency ranges with high modal density and blade-dominated motion. Therefore, a parameter called the selection ratio is introduced here to categorize modes according to how closely they match the assumptions and, thereby, how well they model the system.

One assumption is that the blade motion in the system modes of interest can be represented using a linear combination of cantilevered-blade modes, that is, $\boldsymbol{\Phi}^{s}=\boldsymbol{\Phi}^{\mathrm{cb}} \mathbf{q}$. To check this assumption, the participation of the cantilevered-blade normal mode (s) in the current system normal mode is computed. The participation factor with respect to the stiffness matrix for each blade in the system forms the matrix

$$
\mathbf{q}_{\Phi}^{c b}=(\mathbf{F} \otimes \mathbf{I}) \underset{h=1, \ldots, P}{\tilde{\mathbf{B}} \mathbf{d i a g}}\left[\tilde{\mathbf{q}}_{h}^{\mathrm{cb}}\right]
$$

where $\underset{h=1, \ldots, P}{\tilde{\mathbf{B}} \text { diag }}[\cdot]$ indicates a pseudoblock diagonal matrix, F denotes the real-valued Fourier matrix, and

$$
\tilde{\mathbf{q}}_{h}^{\mathrm{cb}}=\left[\boldsymbol{\Lambda}^{\mathrm{cb}}\right]^{-1}\left(\boldsymbol{\Phi}^{\mathrm{cb}} \mathbf{K}^{\mathrm{cb}} \boldsymbol{\Phi}^{s}\right)
$$

is the participation of the cantilevered-blade normal modes in the cyclic system normal mode for harmonic $h$ [7]. The matrix $\boldsymbol{\Lambda}^{\mathrm{cb}}$ contains the cantilevered-blade eigenvalues and the matrix $\mathbf{K}^{\mathrm{cb}}$ is the stiffness matrix for the cantilevered blade. Large participation factors for each blade indicate that the motion of the blade in the system normal mode is well represented by the motion of the cantileveredblade normal mode and would be an advantageous choice to use in the ROM for the mistuning problem.

A second assumption in the CMM formulation of the mistuning identification problem is that the displacements at the interface between the blade and the disk are small for the system modes used in the ROM. These displacements can be written as $\boldsymbol{\Phi}^{s, \Gamma}$, where $\Gamma$ denotes the boundary between the blades and the disk. If this motion is small relative to the motion of the blades for a given system normal mode, then this mode is a favorable choice for the IROM used for mistuning identification.

Using these two assumptions, a new criterion is formed to effectively evaluate the candidate system normal modes for the IROM used for mistuning identification. This criterion, called the selection ratio (SR), is defined as

$$
\mathrm{SR}_{j}=\frac{\left\|\mathbf{q}_{\Phi, j}^{\mathrm{cb}}\right\|}{\left\|\Phi_{j}^{s, \Gamma}\right\|}
$$

where $j$ denotes the $j$ th system normal mode. This parameter accounts for the two assumptions inherent to the CMM formulation of the mistuning identification problem that have been identified as important for the mistuning identification procedure. The system normal modes with large SR values agree favorably with both of the noted CMM assumptions and, therefore, would be good candidates for the IROM used for mistuning identification.

Although the SR was derived specifically for the CMM approach to mistuning identification, it has a more general interpretation as well. For solving the mistuning identification problem, which is an inverse problem, the ROM should ideally contain only modes that show sensitivity to mistuning. In other words, any mode of the tuned system that would not be changed much by the mistuning is not helpful for solving the mistuning identification problem. Now, consider that the numerator of the SR is related to how strongly a change in a cantilevered-blade eigenvalue (i.e., mistuning) affects the system eigenvalue, and the denominator of the SR is related to the strength of the blade-to-disk (and, thus, blade-to-blade) coupling. Thus, the SR is essentially a mistuning-to-coupling ratio for each system mode. It has been shown by Hodges [28] that the degree of mode localization increases monotonically with an increase in the mistuning-to-coupling ratio. Therefore, the SR is a metric that provides a quantitative assessment of the sensitivity of each system mode to blade mistuning. It is believed that similar metrics could be used with other mistuning identification techniques.

\section{Physical to Modal Transformation}

The CMM approach to mistuning transforms the analysis from physical to modal coordinates to reduce the model size. In general, consider the transformation from physical coordinates $\mathbf{x}$ to modal coordinates $\mathbf{p}$ expressed as

$$
\mathbf{x}=\Phi^{s} \mathbf{p}
$$

To reduce the model size, the matrix of tuned normal mode shapes, $\boldsymbol{\Phi}^{s}$, is truncated. Typically, this truncation simply depends upon the frequency range of interest. The physical coordinates and tuned normal mode shapes are known, and the modal coordinates must first be found from Eq. (16), which is a least-squares problem (because $\boldsymbol{\Phi}^{s}$ is truncated).

Measuring many points per blade is prohibitively expensive. Hence, the mistuning identification procedure is based on experimentally measuring the vibration at only a few points on each blade. These measurement points also correspond to the DOF kept in the modal matrix used for the entire procedure. In this work, the measurement points are chosen using the effective independent distribution vector (EIDV) procedure introduced by Penny et al. [29]. Using a selected basis of tuned-system normal modes, the EIDV algorithm selects DOF from a candidate set that will result in the modes being most distinguishable. It has been shown that the mistuning pattern can be effectively identified using as few as one point per blade [21]. Such a restrictive limit on the number of measured points introduces additional restrictions on the modes that can be used in the IROM. To correctly solve for $\mathbf{p}$ using Eq. (16), the number of measured DOF on the structure must be greater than or equal to the number of tuned-system normal modes. Otherwise, the modal matrix, $\boldsymbol{\Phi}^{s}$, with the reduced number of DOF is rank deficient and that can adversely affect the mistuning identification results.

It should be noted that the modal matrix may become rank deficient even when it has more measurement points than system 
normal modes, that is, in cases in which multiple system normal modes cannot be distinguished with the given set of measurement points. An example of such a situation occurs in the case in which only one point per blade is measured and different tuned-system normal modes having the same number of nodal diameters are kept in the IROM. In this case, only one point is not enough to distinguish the modes with the same nodal diameter content and, therefore, the modal matrix is rank deficient. In such a case, more DOF per blade must be used to achieve a modal matrix with full rank.

\section{Solution of a Known Mistuned Eigenvalue Problem to Generate Numerical Results}

Sections II.B and II.C discussed ways of evaluating tuned-system normal modes that are used as a basis for the mistuning identification procedure. With experience, a modeler could choose an appropriate ROM for the mistuning identification problem. To increase the robustness and reduce the modeling expertise required to build a ROM for performing mistuning identification, a procedure to automatically construct an appropriate IROM is presented next.

Consider Eq. (8). Lim [27] suggested that, if the damping is small and the measurements are taken at resonant frequencies, then $\mathbf{f}^{s}$ and $\gamma$ can be set to zero. This results in

$$
-\omega^{2} \mathbf{p}+\left[\boldsymbol{\Lambda}^{s}+\boldsymbol{\Lambda}^{\delta, s}+\mathbf{q}^{T} \boldsymbol{\Lambda}^{\delta, \mathrm{cb}} \mathbf{q}\right] \mathbf{p}=\mathbf{0}
$$

which can be viewed as an eigenvalue problem with $\omega$ as the eigenvalue and $\mathbf{p}$ as the eigenvector.

Here, it is suggested to first generate a blade stiffness mistuning pattern, $\boldsymbol{\Lambda}_{\text {gen }}^{\delta \text {,cb }}$ and the cyclic model updating pattern, $\boldsymbol{\Lambda}_{\text {gen }}^{\delta, s}$. It should be noted that the normalization of the eigenvalue changes by the nominal eigenvalues typically associated with mistuning has been dropped for convenience. At this point, the eigenvalue problem is solved for $\mathbf{p}$ using the generated mistuning pattern, $\boldsymbol{\Lambda}_{\mathrm{gen}}^{\delta, \mathrm{cb}}$ and $\boldsymbol{\Lambda}_{\mathrm{gen}}^{\delta, s}$. Note that the values of $\mathbf{p}$ must be perturbed to avoid a trivial solution in which any IROM will give accurate results for the mistuning parameters. The solutions, $\omega$ and $\mathbf{p}$, are then used as surrogate data in Eq. (17) (inverse problem) in which the blade stiffness mistuning, $\boldsymbol{\Lambda}^{\delta, \mathrm{cb}}$, and the cyclic model updating, $\boldsymbol{\Lambda}^{\delta, s}$, are no longer considered known. This formulation represents the typical mistuning identification (inverse) problem, which can be solved for the mistuning parameters denoted by $\boldsymbol{\Lambda}_{\text {id }}^{\delta, \text { cb }}$ and $\boldsymbol{\Lambda}_{\text {id }}^{\delta, s}$. Of course, an exact identification gives $\boldsymbol{\Lambda}_{\text {id }}^{\delta, \mathrm{cb}}=\boldsymbol{\Lambda}_{\text {gen }}^{\delta \text {,cb }}$ and $\boldsymbol{\Lambda}_{\text {id }}^{\delta, s}=\boldsymbol{\Lambda}_{\text {gen }}^{\delta, s}$. Here, gen stands for the generated mistuning parameters that are used to solve the direct problem, and id stands for the mistuning parameters identified in the inverse procedure.

With the perturbed values of $\mathbf{p}$, different IROMs can be evaluated by comparing the generated mistuning parameters with those solved by the mistuning identification procedure. By generating a mistuning pattern and solving for the surrogate measurement data as suggested, a mistuning pattern can be identified using the IROM. Then, error metrics can be defined as the difference between the known and the identified values as $\left\|\boldsymbol{\Lambda}_{\text {gen }}^{\delta \text { cb }}-\boldsymbol{\Lambda}_{\text {id }}^{\delta, \text { cb }}\right\|_{2}$ and $\left\|\boldsymbol{\Lambda}_{\text {gen }}^{\delta, s}-\boldsymbol{\Lambda}_{\text {id }}^{\delta, s}\right\|_{2}$ for the blade stiffness mistuning and the cyclic modeling error, respectively. Using these error metrics, the effectiveness of various IROMs for identifying mistuning parameters can be evaluated.

\section{Effect of IROM on Mistuning Parameter Identification}

It is not necessarily simple to select the best IROM for the identification of mistuning parameters. Certain modes are less compatible with the assumptions made in the CMM formulation of the mistuning identification procedure. As mentioned in Sec. II, a method of evaluating the suitability of various modes for the IRŌM has been developed. Also, the limited number of measurement points used for obtaining forced response data (which also corresponds to the DOF used to represent the tuned-system normal modes in the IROM) has a significant impact on which modes should be selected for the mistuning identification procedure.
In this work, we use a 24-bladed disk (shown in Fig. 1) for validations. Only the first flexural cantilevered-blade mode is used in the mistuning procedure, and the candidate tuned-system normal modes come from the $0-5000-\mathrm{Hz}$ frequency range, which envelopes the first flexural blade mode family as can be seen in the frequency vs nodal diameter plot in Fig. 2. The surrogate measurement data used in this section are composed of the vibration response measured at each system resonance for a given frequency sweep. The surrogate measurement data are generated numerically using single-point harmonic excitation applied at blade 1 .

\section{A. Selection Ratio}

In Sec. II, the SR factor was presented to evaluate the tuned-system normal modes that are candidates for the IROM used for mistuning identification. Using a sector of the FEM, the tuned-system normal modes are computed in cyclic coordinates. Using only these modes, the SR values are computed for each mode. The modes are then ranked according to their SR values, with the highest $S R$ value corresponding to the most favorable mode. Thus, IROMs of increasing size can be constructed using this mode ordering. That is, at each iteration, the tuned-system normal mode with the highest available SR value is added to the IROM. Figure 3 shows the mode shapes in the frequency range from 0 to $5000 \mathrm{~Hz}$, which are most closely related to the first flexural cantilevered-blade mode. It can be seen in Figs. $\underline{3 a}$ and $\underline{3 b}$ that the higher nodal diameter modes tend to have higher participation factors and smaller blade-disk interface motion. This indicates that these modes have more blade-dominant motion, whereas modes at lower nodal diameters (in the veering region) have significant disk motion. The blade-dominant modes typically have the largest SR values. These SR values are shown in Fig. 3c, in which the size of each circle denotes the SR value and the numbers indicate the SR-based mode ordering.

Figure $\underline{4}$ depicts the information in Fig. $\underline{3 c}$ in a way that more clearly shows the SR values. In particular, the modes with low SR

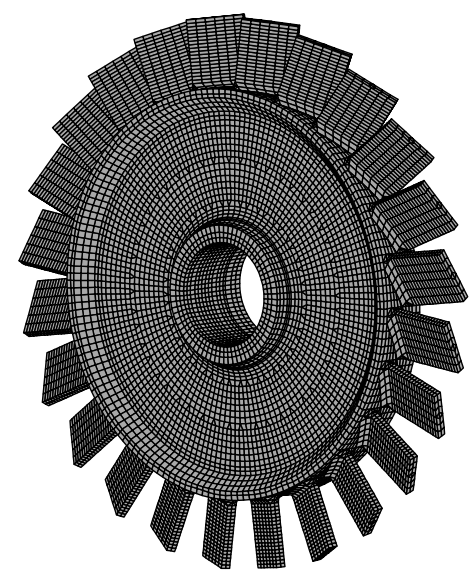

Fig. 1 FEM of the blisk with 24 blades.

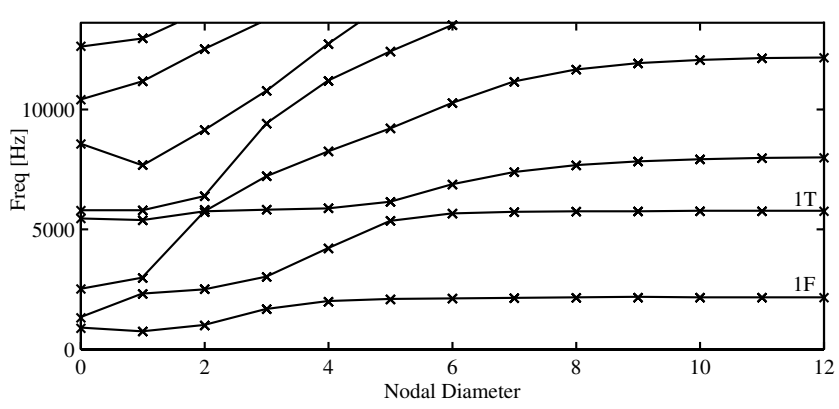

Fig. 2 Natural frequencies vs nodal diameters for the blisk, where 1T refers to the first torsional blade mode family and $1 \mathrm{~F}$ refers to the first flexural blade mode family. 
values, below the dotted line at an SR value of 0.1 , should not be used for constructing the IROM for mistuning identification.

The importance of the IROM construction with respect to yielding accurate mistuning identification results can be seen in Figs. $\underline{5}$ and $\underline{6}$. The measurement data used were from the frequency range of $0-$

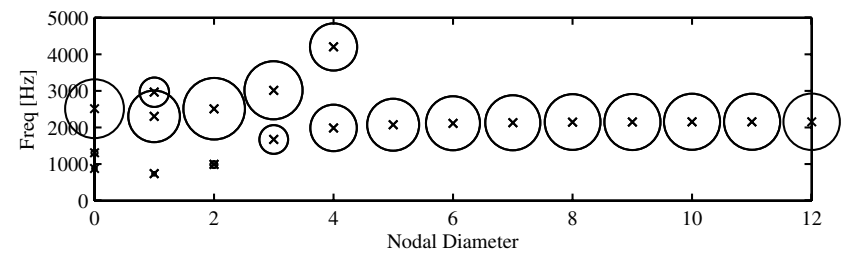

a) Participation factors

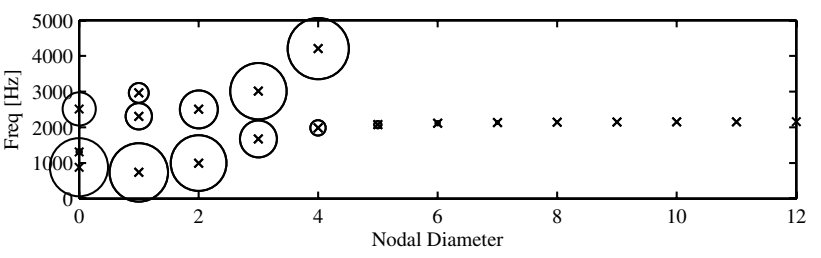

b) Interface motion factors

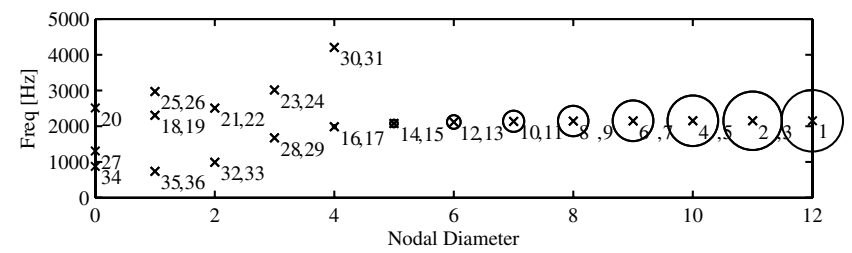

c) Selection ratio (SR) factors. Numbers denote the mode selection order

Fig. 3 Parameters for the IROM selection. The diameter of each circle indicates the magnitude of the parameter.

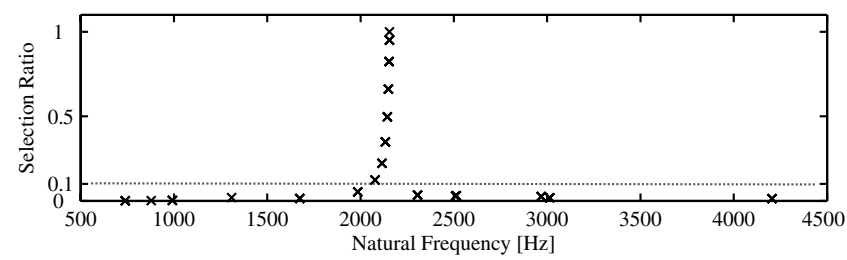

Fig. 4 SR values vs the natural frequency.
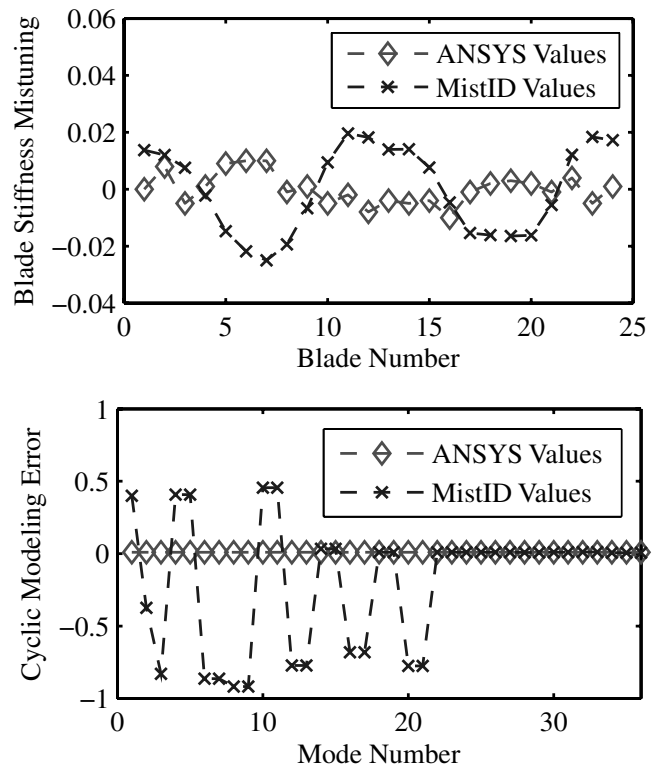

Fig. 5 Parameters for model updating using the 36-DOF IROM (all modes in 0-5000 Hz).
$5000 \mathrm{~Hz}$. The results from the mistuning identification formulation presented in this work (denoted by MistID) are compared with values computed using ANSYS. Figure 5 shows the blade stiffness mistuning, $\boldsymbol{\Lambda}^{\delta, \mathrm{cb}}$, and cyclic modeling error, $\boldsymbol{\Lambda}^{\delta, s}$, in the case in which all of the modes in the $0-5000-\mathrm{Hz}$ frequency range are used in the IROM. Figure 5a shows that the general pattern of mistuning is not captured. Similarly, the cyclic modeling error values shown in Fig. $\underline{5 b}$ exhibit extremely large errors compared with their exact value of 0.01 .

Figure $\underline{6}$ shows the mistuning identification results using a model that contains only the 15 modes that have SR values above the threshold value of 0.1 (see Fig. 4). It is clear that the results for the both blade stiffness mistuning shown in Fig. 6a and cyclic modeling error shown in Fig. 6b have improved significantly from those shown in Fig. 5. These results indicate that the automatic mode selection based on the SR values performed well in this case.

It should also be noted that the SR values provide useful information about the tuned-system modes in general. Under the assumption that the blade motion in the system modes of interest can be represented using a cantilevered-blade mode or a combination of a few modes, one can determine to which family of blade modes the system mode belongs. In Fig. 3a, the cantilevered-blade mode used to compute the participation factors was the first flexural blade mode. Therefore, the modes that belong to the first flexural blade mode family are shown by larger circles in Fig. 3c. The interface motion in Fig. 3 b helps to adjust the selection ratio to show the modes that most closely fit in that blade mode family.

\section{B. Restriction on Nodal Diameter Representation Based on Limited Measurement Points}

One key consideration when choosing the IROM for mistuning identification is the rank of the modal matrix containing the tunedsystem normal modes. This matrix can be rank deficient because only DOF physically measured on the structure are used to represent the mode shapes. For example, the matrix can become rank deficient when trying to distinguish between modes having the same nodal diameter when too few measurement points are used.

Figure 7 depicts the selection order of the mode shapes represented using only one measurement point per blade contained in the 1900 $5000-\mathrm{Hz}$ frequency range. The ordering of these modes is based strictly upon the SR values. It should be noted that the 23rd and 24th modes are the second mode pair added for the first nodal diameter. However, it is not until the 25th and 26th modes that the third nodal diameter is represented.
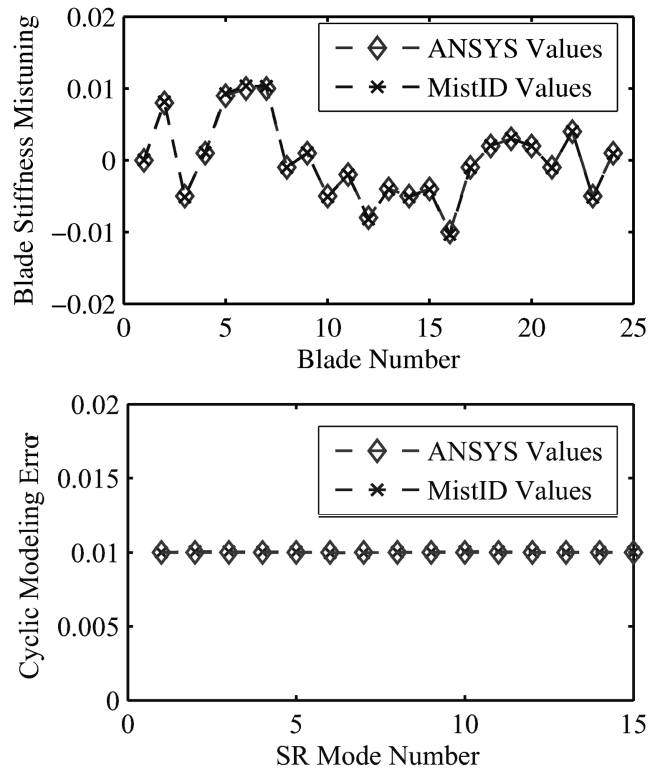

Fig. 6 Parameters for model updating using the 15-DOF IROM (modes in 0-5000 $\mathrm{Hz}$ with an SR value above 0.1 ). 


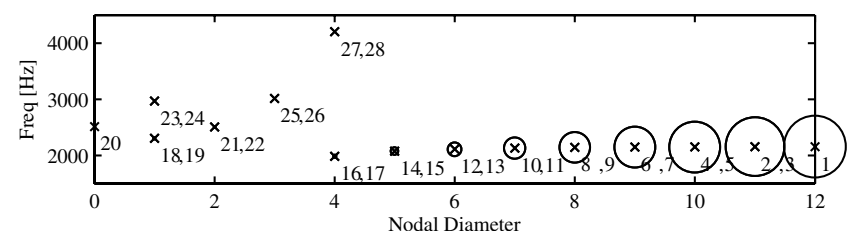

Fig. 7 Mode ordering based strictly on the SR values.

In contrast to Fig. 7, the plot in Fig. 8 shows the ordering of the tuned-system normal modes based upon the SR values with the exception of the modes that would cause duplicity of the mode or mode pair for a given nodal diameter. Any mode or mode pair that would be the second for a given nodal diameter is not added until each nodal diameter has been represented by a mode or mode pair. As opposed to the ordering in Fig. 7, the ordering in Fig. 8 for the 23rd and 24 th modes has changed to be at nodal diameter $\overline{3}$, which was previously only represented with the 25 th and 26 th modes.

The rank of the modal matrix, given one measurement DOF per blade, is tracked in Fig. 9a as it is built up using the mode shapes ordered as shown in Fig. 7. Upon the addition of the 23rd mode to the IROM, the modal matrix becomes rank deficient. However, in Fig. 9b, in which two measurement DOF per blade are used, the modal matrix does not become rank deficient.

The benefit of this selection procedure is that, with one measurement point per blade ( 24 total measurement points), modes with differing nodal diameter content are far more distinguishable and, therefore, will not result in a rank deficient matrix. This is shown in Fig. 10a in which it is clear that the rank deficiency occurs at mode $2 \overline{5}$, which is the first mode added that duplicates a nodal diameter. Using the algorithm that ensures that all nodal diameter

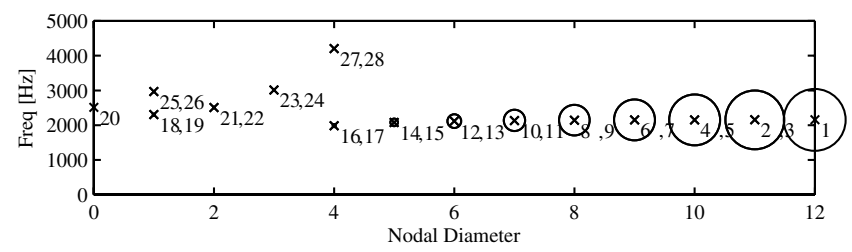

Fig. 8 Mode ordering based on both the SR values and the nodal diameter content.

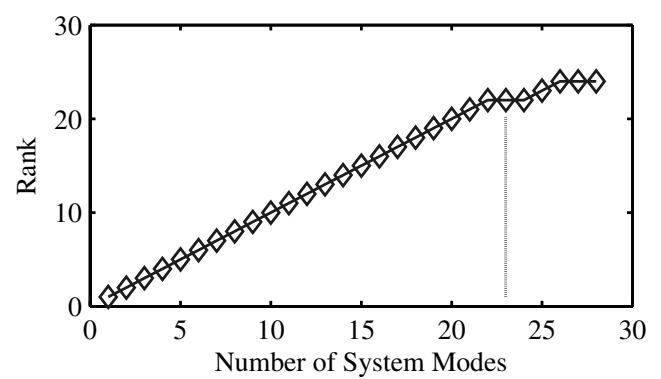

a) One measurement DOF per blade

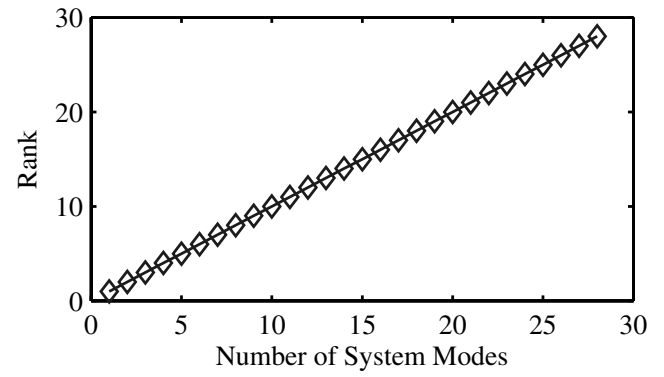

b) Two measurement DOF per blade

Fig. 9 Rank of the modal matrix as the IROM is built up by adding modes based strictly on the SR values. content is represented before duplicating nodal diameter modal content is recommended because of the limited number of DOF used to represent the mode shape. It can be seen in Fig. $10 \mathrm{~b}$ that using a second measurement DOF per blade generates a modal matrix with full rank. In general, the restriction of the nodal diameter content of modes can be eliminated by measuring more DOF per blade than the number of modes or mode pairs of a given nodal diameter that will be used in the IROM.

\section{Evaluation of the Inverse ROM by Prescribing Surrogate Data}

The results in Secs. III.A and III.B show that IROM selection can be improved using the ideas presented in Secs. II.A-II.C. However, an arbitrary lower threshold value of 0.1 for the SR values was used to determine the size of the IROM. This requires experience to generate an input to the procedure. Following the analysis presented in Sec. II.D, a more systematic and automatic method for selecting the IROM size is further examined.

Figure 11 shows a flowchart of the procedure that makes use of the analysis of Sec. II.D. The first step involves generating (i.e., prescribing) a mistuning pattern. It would generally be advisable to select a random mistuning pattern that has roughly the same level of mistuning that is expected to most effectively evaluate the IROMs used for mistuning identification. At this point, it is important to distinguish between a ROM used to solve the direct problem (direct ROM or DROM) and a ROM used to solve the inverse problem (inverse ROM or IROM). The accuracy of the DROM with respect to the parent FEM increases monotonically as modes are added. Therefore, a DROM constructed from all the available modes in a given frequency range can be used in place of the FEM for solving the direct problem and generating the test data for a prescribed mistuning pattern. In contrast, the accuracy of the IROM does not increase monotonically as modes are added due to the nature of the leastsquares approximations used in Eq. (16) and in the solution of the inverse identification problem.

The generated/prescribed mistuning pattern in which all of the modes for a given frequency range are employed is then plugged into the DROM. The governing equation for the DROM can be viewed as an eigenvalue problem for which the eigenvalue is $\omega$ and the eigenvector is $\mathbf{p}$. Solving for the eigenvalue and eigenvector yields preliminary surrogate data. Before these data can be used as

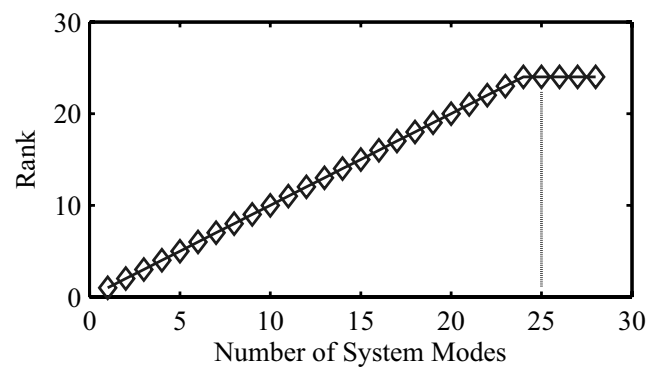

a) One measurement DOF per blade

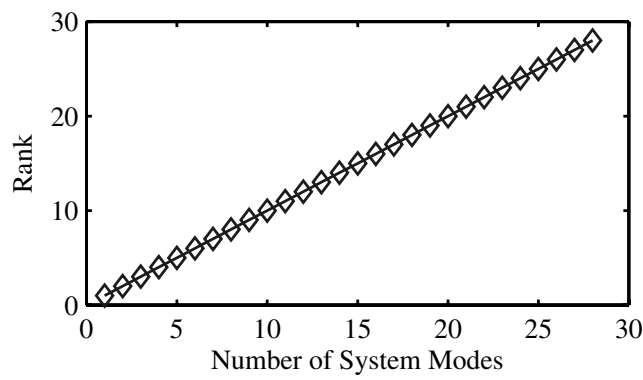

b) Two measurement DOF per blade

Fig. 10 Rank of the modal matrix as the IROM is built up by adding modes based on both the SR values and the nodal diameter content. 


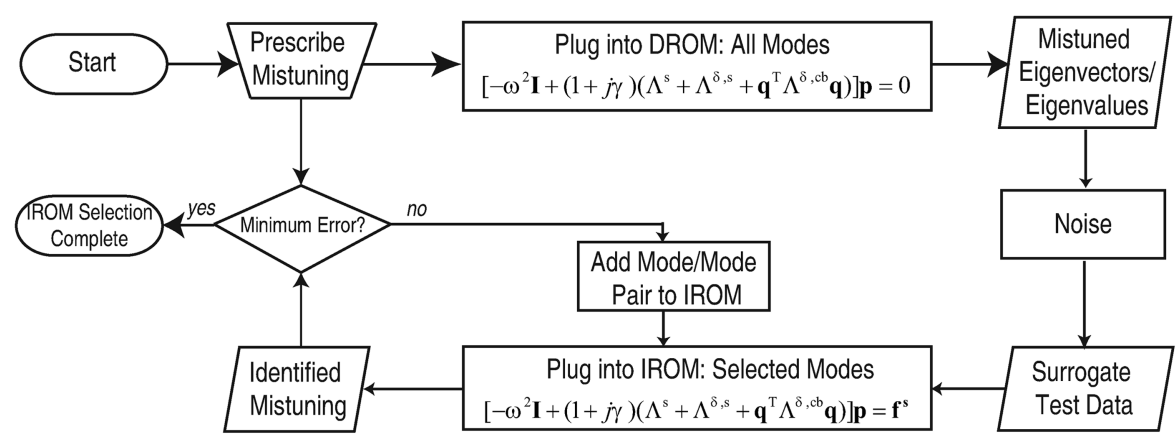

Fig. 11 Flow diagram for the IROM evaluation using surrogate data.

surrogate measurement data, the eigenvector is perturbed. This avoids the trivial solution when the inverse problem is solved. If the data generated are unperturbed, all possible IROMs will yield nearly the same results and the identified mistuning will match the generated mistuning with negligible error. Once the noise is added, the surrogate test data set is formed of the perturbed eigenvectors and unperturbed eigenvalues of the DROM. This surrogate data is then plugged into the IROM, for which the mistuning parameters are unknown. The IROM is made of a subset of the modes used in the DROM. Using the IROM with the surrogate data, a mistuning pattern can be computed. This mistuning pattern can be compared with the generated/prescribed mistuning pattern using the error presented in Sec. II.D. After the error is evaluated, additional modes are added to the IROM and another mistuning pattern is generated. The new resulting error can be computed. If the error reaches a minimum or a satisfactory level, the IROM selection is complete. This IROM can then be trusted to identify mistuning from real measured data. In this manner, the performance of various IROMs can be evaluated quantitatively while working only within the reduced-order modeling framework, and the IROM construction procedure can be fully automated.

In this section, the frequency range for possible mode shapes is 0 $5000 \mathrm{~Hz}$. The frequency range for the measurement data is 1900$2160 \mathrm{~Hz}$. Figure 12 shows the absolute error results obtained by comparison of the known mistuning from the FEM and the mistuning values generated using the mistuning identification procedure. First, the benefit of using the SR-based mode ordering is clear, as both the stiffness mistuning and cyclic modeling error have a smaller absolute error when the SR values are used. The detailed view of the stiffness mistuning in Fig. 12a shows that the error is suitably low in the range of 15-24 modes. However, the goal is to be able to predict what IROM will produce a low absolute error without knowing the solution from a FEM.

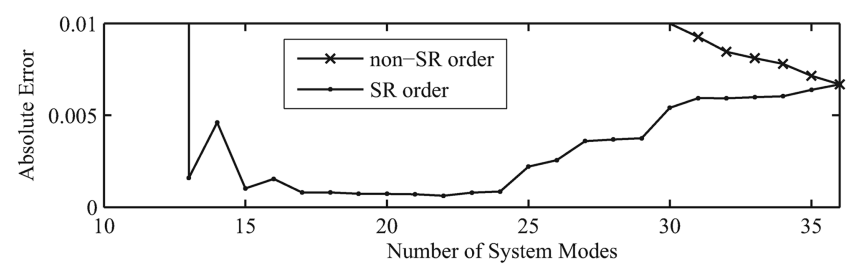

a) Absolute error of blade stiffness mistuning

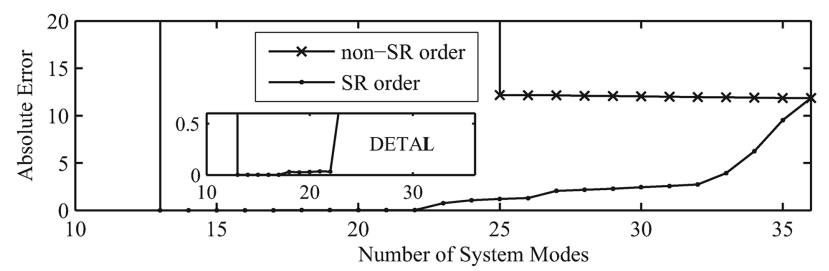

b) Absolute error of cyclic modeling error

Fig. 12 Absolute error of the model updating parameters in comparison with known FEM values with identified values obtained by adding candidate modes in the $0-5000-\mathrm{Hz}$ range.
For the direct problem, the level of perturbation to the eigenvectors $\mathbf{p}$ to produce the surrogate data was $\pm 1 \%$ of $\mathbf{p}$. Figure 13 shows the absolute error results obtained by comparison of the known mistuning by solving the direct problem with a larger DROM and a prescribed mistuning pattern as detailed in this section and the mistuning values generated using the mistuning identification procedure. From the plots in Fig. 13, it is evident that the general trends of absolute error can be predicted using a larger DROM for reference. According to Fig. 13a, a relatively low absolute error occurs for SR-ordered IROMs of size 13-19. Figure 14 shows the absolute errors for the direct problem, but with additional information about the blades. The sensitivity to modeling errors in the inverse problem is not uniform across all blades. Therefore, it is possible for the error to be affected by the mistuning

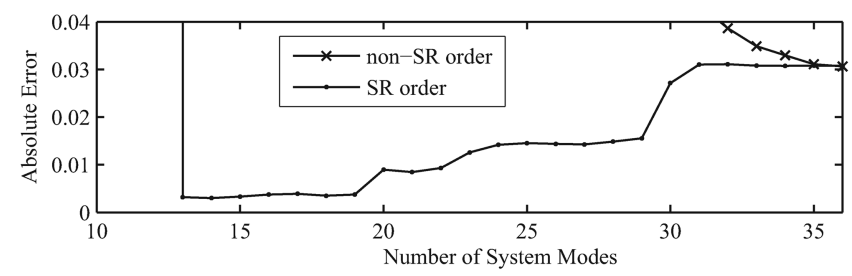

a) Absolute error of blade stiffness mistuning

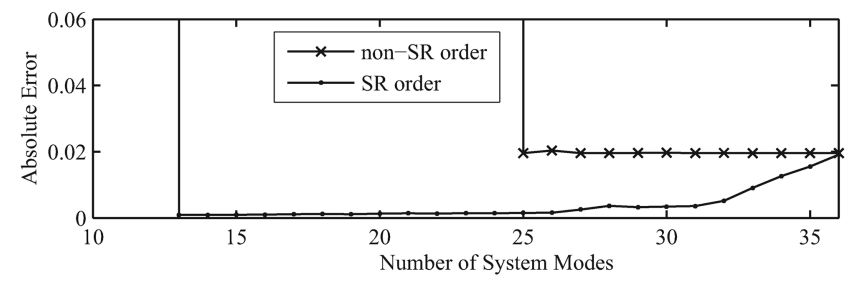

b) Absolute error of cyclic modeling error

Fig. 13 Absolute error of the model updating parameters in comparison with known values obtained using a DROM with identified values obtained by adding candidate modes in the $0-5000-\mathrm{Hz}$ range.

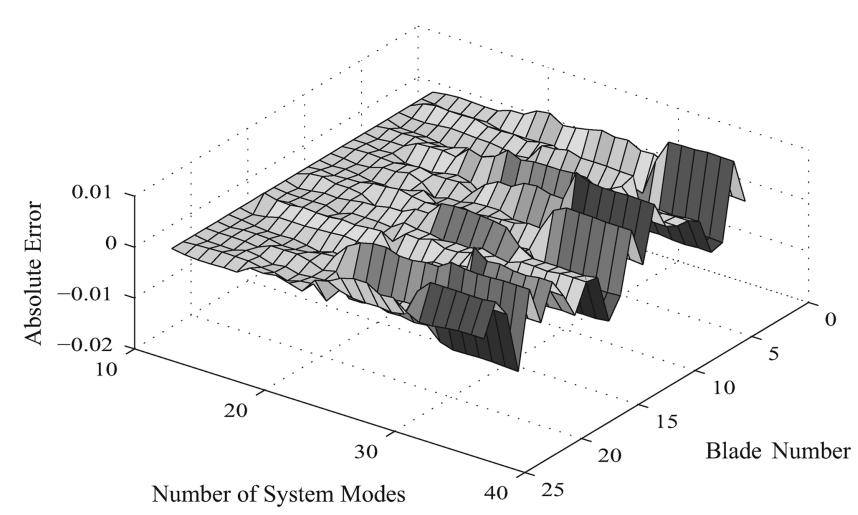

Fig. 14 Absolute error of blade stiffness mistuning across 24 blades. 
pattern. However, we observed that in general the error does not change significantly. This predicts that the selected IROM is a good choice for the mistuning identification procedure with respect to the stiffness mistuning. Similar to the plot in Fig. 12b, Fig. 13b shows steadily increasing values of the absolute error. Therefore, these results indicate that the error trends could be used to determine an IROM size that yields robust and accurate mistuning identification results.

\section{Conclusions}

Techniques to more effectively identify the mistuning parameters of blisks using the CMM approach to mistuning were presented. A quantitative metric, the SR value, was introduced to systematically evaluate the tuned-system modes used for forming the IROM in the CMM approach to mistuning identification. The SR values take into account the two assumptions made in the CMM technique, namely, that the cantilevered-blade normal mode shapes are similar to the blade portion tuned-system normal mode shapes and that the bladedisk interface motion is small compared with the cantilevered-blade motion. The SR values are generated using only information from the tuned-system normal modes generated from the tuned FEM. Using these SR values, an effective IROM can be formed using tunedsystem normal modes. Using IROMs without ordering modes according to SR values can result in inaccurate mistuning identification. For these same cases, ordering modes according to the SR values leads to accurate mistuning parameters. Therefore, using this metric to select modes for the IROM can dramatically improve the accuracy of the mistuning identification results. It was also noted that these SR values could be used to categorize modes in a quantitative fashion according to blade mode families.

The effect of using a limited number of measurement points to represent the forced response and modes shapes was also considered. Because the current mistuning identification formulation is intended for use with experimental data, tuned-system mode shapes are represented with a reduced number of DOF. This places a limitation on the number and type of tuned-system modes that can be used in the IROM for mistuning identification. If there are not enough measurement points, the reduced modal matrix is rank deficient. An important example of such rank deficiency occurs when representing modes have the same nodal diameter content, as demonstrated in this paper. To use multiple modes with the same nodal diameter content, it was shown to be necessary to use more measurement DOF per blade than the number of modes (in the ROM) that have the same nodal diameter.

In addition, a novel technique has been developed to automatically determine a suitable IROM size for solving the mistuning identification inverse problem. The approach is to prescribe a blade mistuning pattern and compute surrogate measurement data numerically using a larger ROM for the direct problem. These measurement data are produced by solving the forward problem and then perturbing the solution before plugging it into the inverse solver. The inverse formulation assumes that the mistuning is unknown and the IROM used is a subset of the ROM used for the direct problem. The solution to the inverse problem can then be compared directly to the prescribed mistuning pattern to check the performance of the IROM as modes are added. The results presented show that this process enables the automated construction of an IROM and, thus, improves the overall accuracy and robustness of the mistuning identification. Furthermore, the automation of the procedure guarantees a systematic identification that does not demand the expertise that other current procedures require.

\section{Acknowledgments}

This work was supported by GE Aviation through the University Strategic Alliance (USA) program, with Sergio Filippi and Steve Manwaring as the technical contacts and Dave Wisler as the program manager. In this paper, there are no data representative of an actual GE blisk. We would like to especially thank Sergio Filippi for his fruitful conversations and suggestions regarding this research.

\section{References}

[1] Thomson, D. E., and Griffin, J. T., "The National Turbine Engine High Cycle Fatigue Program," Global Gas Turbine News, Vol. 39, No. 1, 1999, pp. 14-17.

[2] Castanier, M. P., Óttarsson, G., and Pierre, C., "A Reduced-Order Modeling Technique for Mistuned Bladed Disks," Journal of Vibration and Acoustics, Vol. 119, No. 3, 1997, pp. 439-447. doi:10.1115/1.2889743

[3] Bladh, R., Castanier, M. P., and Pierre, C., "Component-Mode-Based Reduced Order Modeling Techniques for Mistuned Bladed DisksPart 1: Theoretical Models," Journal of Engineering for Gas Turbines and Power, Vol. 123, No. 1, 2001, pp. 89-99. doi: $10.1115 / 1.1338947$

[4] Bladh, R., Castanier, M. P., and Pierre, C., "Component-Mode-Based Reduced Order Modeling Techniques for Mistuned Bladed DisksPart 2: Application," Journal of Engineering for Gas Turbines and Power, Vol. 123, No. 1, 2001, pp. 100-108. doi:10.1115/1.1338948

[5] Yang, M.-T., and Griffin, J. H., "A Reduced-Order Model of Mistuning Using A Subset of Nominal System Modes," Journal of Engineering for Gas Turbines and Power, Vol. 123, No. 4, 2001, pp. 893-900. doi:10.1115/1.1385197

[6] Feiner, D. M., and Griffin, J. H., "A Fundamental Model of Mistuning for a Single Family of Modes," Journal of Turbomachinery, Vol. 124, No. 4, 2002, pp. 597-605. doi: $10.1115 / 1.1508384$

[7] Lim, S., Bladh, R., Castanier, M. P., and Pierre, C., "A Compact, Generalized Component Mode Mistuning Representation for Modeling Bladed Disk Vibration," Collection of Technical Papers-AIAA/ ASME/ASCE/AHS/ASC Structures, Structural Dynamics and Materials Conference, Vol. 2, AIAA, Reston, VA, 2003, pp. 1359-1380.

[8] Lim, S., Bladh, R., Castanier, M. P., and Pierre, C., "Compact, Generalized Component Mode Mistuning Representation for Modeling Bladed Disk Vibration," AIAA Journal, Vol. 45, No. 9, 2007, pp. 22852298.

doi: $10.2514 / 1.13172$

[9] Mignolet, M. P., and Lin, C.-C., "Identification of Structural Parameters in Mistuned Bladed Disks," Journal of Vibration and Acoustics, Vol. 119, No. 3, 1997, pp. 428-438. doi:10.1115/1.2889742

[10] Pichot, F., Thouverez, F., Jezequel, L., and Seinturier, E., "Mistuning Parameters Identification of a Bladed Disk," Key Engineering Materials, Vols. 204-205, 2001, pp. 123-132.

[11] Judge, J., Pierre, C., and Ceccio, S. L., "Experimental Identification of Mistuning in Blisks," Proceedings of the 6th National Turbine Engine High Cycle Fatigue Conference, Universal Technology Corporation, Dayton, OH, 2001.

[12] Judge, J., Pierre, C., and Ceccio, S. L., "Experimental Validation of Mistuning Identification Techniques and Vibration Predictions in Bladed Disks," Proceedings of the International Forum on Aeroelasticity and Structural Dynamics, Vol. 2, Asociación de Ingenieros Aeronáuticos de España, Madrid, 2001, pp. 89-98.

[13] Judge, J. A., Pierre, C., and Ceccio, S. L., "Mistuning Identification in Bladed Disks," Proceedings of the International Conference on Structural Dynamics Modelling, Instituto Superior Tecnico, Lisbon, 2002.

[14] Pierre, C., Judge, J., Ceccio, S. L., and Castanier, M. P., "Experimental Investigation of the Effects of Random and Intentional Mistuning on the Vibration of Bladed Disks," Proceedings of the 7th National Turbine Engine High Cycle Fatigue Conference, Universal Technology Corporation, Dayton, OH, 2002.

[15] Feiner, D. M., and Griffin, J., "A Completely Experimental Method of Mistuning Identification in Integrally Bladed Rotors," Proceedings of the 8th National Turbine Engine High Cycle Fatigue Conference, Universal Technology Corporation, Dayton, OH, 2003, pp. 1-1-1-13.

[16] Kim, N. E., and Griffin, J., "System Identification in Higher Modal Density Regions of Bladed Disks," Proceedings of the 8th National Turbine Engine High Cycle Fatigue Conference, Universal Technology Corporation, Dayton, OH, 2003, pp. 1-68-1-82.

[17] Feiner, D., and Griffin, J., "Mistuning Identification of Bladed Disks Using a Fundamental Mistuning Model-Part 1: Theory," Journal of Turbomachinery, Vol. 126, No. 1, 2004, pp. 150-158. doi: $10.1115 / 1.1643913$

[18] Feiner, D., and Griffin, J., "Mistuning Identification of Bladed Disks Using a Fundamental Mistuning Model—Part 2: Application," Journal of Turbomachinery, Vol. 126, No. 1, 2004, pp. 159-165. doi: $10.1115 / 1.1643914$

[19] Lim, S., Castanier, M. P., and Pierre, C., "Mistuning Identification and 
Reduced-Order Model Updating for Bladed Disks Based on a Component Mode Mistuning Technique," Proceedings of the 9th National Turbine Engine High Cycle Fatigue Conference, Universal Technology Corporation, Dayton, OH, 2004.

[20] Li, J., Pierre, C., and Ceccio, S. L., "Validation of a New Technique for Mistuning Identification and Model Updating Based on Experimental Results for an Advanced Bladed Disk Prototype," Evaluation, Control and Prevention of High Cycle Fatigue in Gas Turbine Engines for Land, Sea and Air Vehicles (Meeting Proceedings RTO-MP-AVT-121), NATO Research and Technology Organization, Neuilly-sur-Seine, France, 2005, pp. 36-1-36-16.

[21] Song, S. H., Castanier, M. P., and Pierre, C., "System Identification of Multistage Turbine Engine Rotors," American Society of Mechanical Engineers Paper GT2007-28307, New York, 2007.

[22] Pichot, F., Laxalde, D., Sinou, J. J., Thouverez, F., and Lombard, J. P., "Mistuning Identification for Industrial Blisks Based on the Best Achievable Eigenvector," Computers and Structures, Vol. 84, Nos. 2930, 2006, pp. 2033-2049. doi:10.1016/j.compstruc.2006.08.022

[23] Lim, T. W., and Kashangaki, T. A. L., "Structural Damage Detection of Space Truss Structures Using Best Achievable Eigenvectors," AIAA Journal, Vol. 32, No. 5, 1994, pp. 1049-1057. doi: $10.2514 / 3.12093$

[24] Hurty, W. C., "Dynamic Analysis of Structural Systems Using
Component Modes," AIAA Journal, Vol. 3, No. 4, 1965, pp. 678-685. doi: $10.2514 / 3.2947$

[25] Craig, R. R., Jr., and Bampton, M. C. C., "Coupling of Substructures for Dynamic Analyses," AIAA Journal, Vol. 6, No. 7, 1968, pp. 13131319.

doi: $10.2514 / 3.4741$

[26] Yang, M.-T., and Griffin, J. H., "A Normalized Modal Eigenvalue Approach for Resolving Modal Interaction," Journal of Engineering for Gas Turbines and Power, Vol. 119, No. 3, 1997, pp. 647-650. doi:10.1115/1.2817033

[27] Lim, S.-H., "Dynamic Analysis and Design Strategies for Mistuned Bladed Disks," Ph.D. Thesis, The University of Michigan, Ann Arbor, MI, April 2005.

[28] Hodges, C. H., "Confinement of Vibration by Structural Irregularity," Journal of Sound and Vibration, Vol. 82, No. 3, 1982, pp. 411-424. doi:10.1016/S0022-460X(82)80022-9

[29] Penny, J. E. T., Friswell, M. I., and Garvey, S. D., "Automatic Choice of Measurement Locations for Dynamic Testing," AIAA Journal, Vol. 32, No. 2, 1994, pp. 407-414.

doi: $10.2514 / 3.11998$
R. Ohayon

Associate Editor 